\title{
Defocus Study of a Novel Optical Antenna Illuminated by a Radial Radiation Fiber Laser
}

\author{
Ping Jiang ${ }^{1 *}$, Huajun $\mathrm{Yang}^{1}$, Kang $\mathrm{Xie}^{2}$, Mingyin $\mathrm{Yu}^{1}$, and Shengqian Mao ${ }^{1}$ \\ ${ }^{I}$ College of Physical Electronics, University of Electronic Science and Technology of China, \\ Sichuan Province 610054, China \\ ${ }^{2}$ College of Instrument Science and Opto-electronic Engineering, Hefei University of Technology, \\ Hefei 230009, China
}

(Received July 4, 2014 : revised August 13, 2014 : accepted August 18, 2014)

\begin{abstract}
A novel antenna with ellipsoid-paraboloid surfaces configuration is designed for matching the incident radial radiation fiber laser distribution for maximum transmission efficiency. The on-axial and off-axial defocus effects on the optical antenna system, resulting in energy loss, are analyzed in detail. Knowledge of the effects of those defocuses on beam divergence, aberration and antenna transmission efficiency is of great importance to the long range communication systems.
\end{abstract}

\author{
Keywords : Radial radiation fiber laser, Ellipsoid-paraboloid surfaces, On-axial defocus, Off-axial \\ defocus, Receiving efficiency \\ OCIS codes : (080.2740) Geometric optical design; (060.4510) Optical communications; (120.1680) \\ Collimation; (140.3510) Lasers, fiber
}

\section{INTRODUCTION}

Laser communications and radar systems employ conventional telescopes as optical antennas. These telescopes, such as Cassegrain telescopes, are always illuminated by an on-axial emission semiconductor laser and frequently have reflection caused by the secondary mirror center is shown in Fig. 1(a). Intensity patterns of an input Gaussian laser beam and truncated Gaussian beam emitted from the antenna are given in Fig. 1(b) and (c), respectively. Because of the on-axial transmission, energy loss caused by the secondary mirror center reflection will greatly depress the emission efficiency in the optical communication system [1-3].

In recent years, fiber lasers attract very much attention $[4,5]$. They are capable of producing stable,high power, allow design freedom, and produce good coherence radiation. They may prove to be an important new light source for applications in medical imaging, sensing, bio-sensing and optical communication systems[6, 7]. In April 2012, an important development in this area: a radial radiation laser using a hollow-core Bragg fiber in combination with organic dye-doped water plugs placed inside the fiber core, was reported in Nature Photonics[8]. This kind of fiber laser is on-axial pumped, resulting in a unique radiating field pattern characterized by cylindrical symmetry and a fixed polarization pointed in the azimuthal direction[9]. This new capability may prove to be an important new light source for applications in space optical communication systems.

In this paper, a novel optical antenna with ellipsoid-paraboloid mirror surfaces is constructed, which is assumed to be illuminated by a radial radiation fiber laser (as shown in Fig. 2(a)), producing stable, on-axially symmetric ring-like radiation (as shown in Fig. 2(b)).

By geometrical optical theory, the design details are proposed and the resultant curves that display the energy loss of the communication system due to defocus between primary and secondary mirrors of emitting antenna are presented. This new type of antenna illuminated by a radial radiation fiber laser, which produces a hollow beam of high precision collimation, may effectively avoid the energy loss caused by the secondary mirror central reflection in the traditional on-axial propagation optical antenna. The investigation results will offer fundamental research for the collimation accuracy and propagation efficiency enhancement of the free space optical communication.

\footnotetext{
*Corresponding author: jiangp@uestc.edu.cn

Color versions of one or more of the figures in this paper are available online.
} 


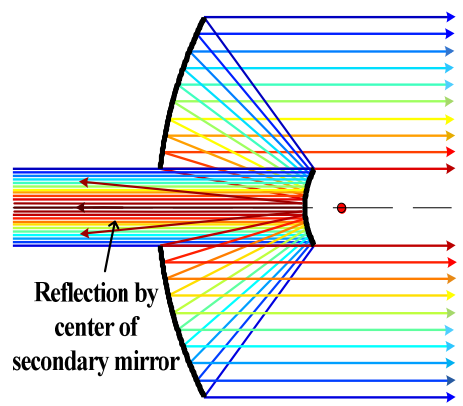

(a)

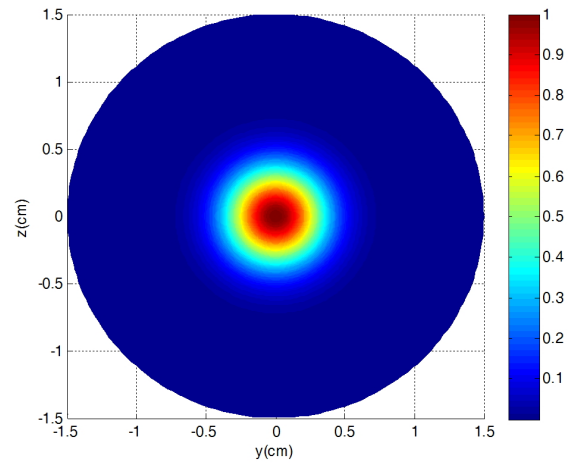

(b)

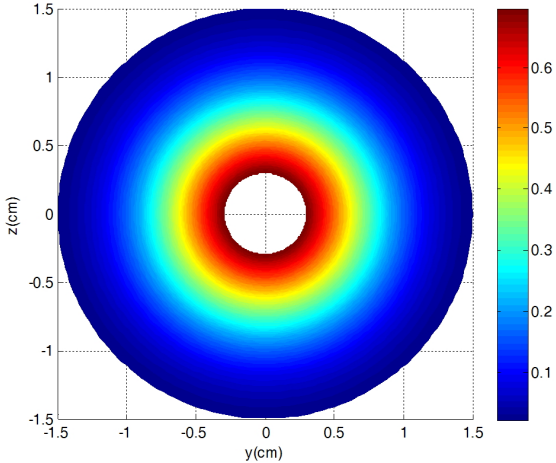

(c)

FIG. 1. Model of Cassegrain antenna and energy distributions. (a) The schematic illustration of central reflection caused by the secondary mirror, (b) energy distribution of the input Gaussian beam, (c) energy distribution of the truncated Gaussian beam emits from antenna.

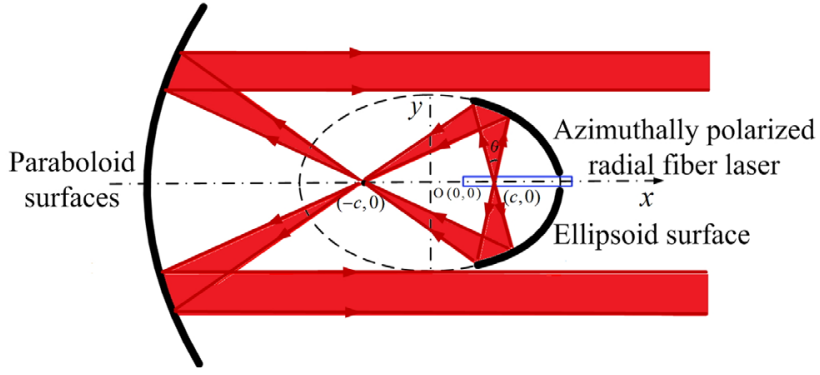

(a)

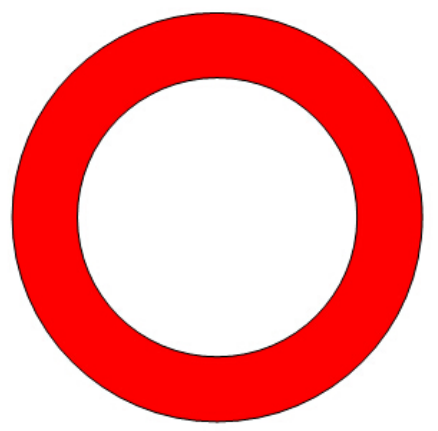

(b)

FIG. 2. Schematic illustration of novel optical emitting antenna illuminated by a radial radiation fiber laser. (a) Configuration of emitting antenna with ellipsoid-paraboloid mirror surfaces and (b) the cross section of the emitting laser beam.

\section{DESIGN OF THE ELLIPSOID-PARABOLOID SURFACES ANTENNA}

Figure 3 shows the two-dimensional structure model of the ellipsoid mirror surface in the $x y$ plane, which performs as a secondary mirror, and is illuminated by a radial radiation fiber laser. The center of the ellipse is at origin point $O(0,0)$. The transmission axis of the pump light, which is

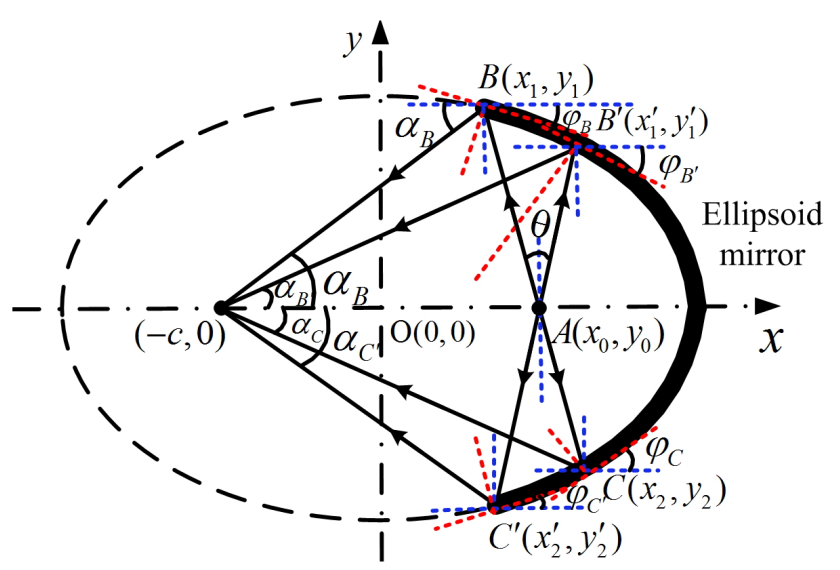

FIG. 3. Two-dimensional structure of the ellipsoid mirror.

along the fiber core, points in the $x$-axis direction. The radial radiation that surrounds the fiber on-axially symmetric leads to an azimuthally divergent angle labeled $\theta$ in the $x y$ plane. By using the geometrical optical theory, here we directly image the laser light at the $x y$ plane.

The radial emitting region of the fiber laser, which can be regarded as a point course, is located at the right side focus of the ellipse, its coordinate is $A\left(x_{0}, y_{0}\right)$, where $x_{0}=c$, $y_{0}=0, c=\sqrt{a^{2}-b^{2}}, a$ and $b$ represent the lengths of long half-axis and short half-axis of the ellipse, respectively. The equations of emitting light $\overline{B C}$ and the ellipse reflector as shown below

$$
\left\{\begin{array}{l}
y-y_{0}=k\left(x-x_{0}\right) \\
\frac{x^{2}}{a^{2}}+\frac{y^{2}}{b^{2}}=1
\end{array}\right.
$$

where $k=\operatorname{tg}\left(90^{\circ}-\frac{\theta}{2}\right)=\operatorname{ctg} \frac{\theta}{2}$ is the slope of the emitting light $\overline{B C}$. 
Two sets of solutions $\left\{\begin{array}{l}B\left(x_{1} y_{1}\right) \\ C\left(x_{2} y_{2}\right)\end{array}\right.$, derived by solving Eq. (1), represent two intersections of emitting light $\overline{B C}$ and the ellipsoid mirror surface.

By deriving the ellipse equation the slope of the tangent at point $B\left(x_{1}, y_{1}\right)$ can be obtained as $\tan \varphi_{B}=\left.\frac{d y}{d x}\right|_{B\left(x_{1}, y_{1}\right)}=$ $-\frac{b^{2} x_{1}}{a^{2} y_{1}}$.

The slope angle of reflecting light $\overline{B D}$ at point $B\left(x_{1}, y_{1}\right)$ can be written as $\alpha_{B}=90+2 \varphi_{B}+\frac{\theta}{2}$.

The two-dimensional structure of the ellipsoid-paraboloid mirror surfaces is shown in Fig. 4. The equations of reflecting light $\overline{B D}$ and the paraboloid mirror, which performs as a primary mirror of the emitting antenna, is shown below

$$
\left\{\begin{array}{l}
\left(y-y_{p}\right)^{2}=2 p\left(x-x_{p}\right) \\
y-y_{1}=k^{\prime \prime}\left(x-x_{1}\right)
\end{array}\right.
$$

where $\left.k^{\prime \prime}\right|_{B\left(x_{1}, y_{1}\right)} k^{\prime \prime} \mid \tan \alpha_{B}$ is the slope of the reflecting light $\overline{B D}$, point $p\left(x_{p}, y_{p}\right)$ is the apex of the parabola. Solution $D\left(x_{3}, y_{3}\right)$ derived by solving the Eq. (2) represents the intersection coordinate of reflected light $\overline{B D}$ and the paraboloid mirror. By deriving the parabola equation the slope of the tangent at point $D\left(x_{3}, y_{3}\right)$ can be obtained as $\operatorname{tg} \eta_{D}=\left.\frac{\mathrm{d} y}{\mathrm{~d} x}\right|_{D\left(x_{3}, y_{3}\right)}=\frac{p}{\left(y_{3}-y_{5}\right)}$

The divergence angle of the outer edge reflecting light below the $x$ axis (labeled 1 in Fig. 4) is

$$
\beta_{1}=180^{\circ}+2 \eta_{D}-\alpha_{B}
$$

The divergence angle of the outer edge reflecting light above the $x$ axis (labeled $1^{\prime}$ in Fig. 4) can be written as

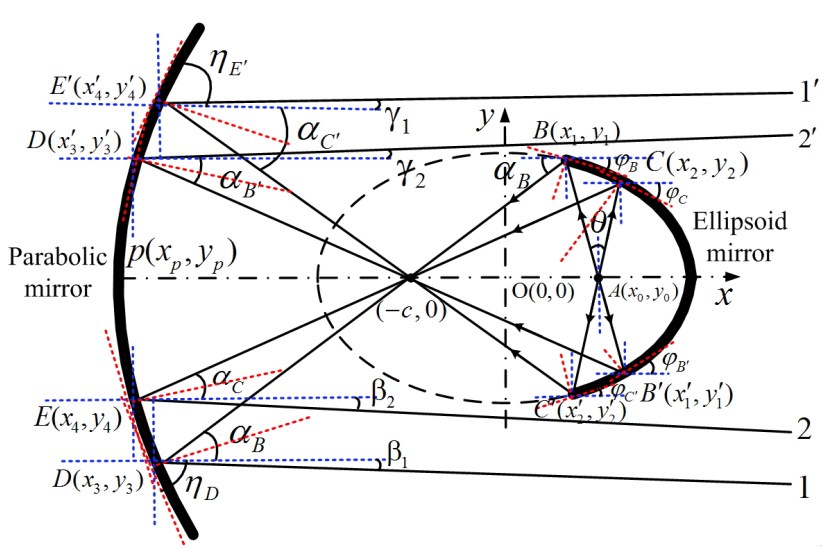

FIG. 4. Two-dimensional structure of the ellipsoid-paraboloid mirrors.

$$
\gamma_{1}=2 \eta_{E^{\prime}}-\alpha_{C^{\prime}}
$$

The divergence angles of inner edge reflecting light below and above the $x$ axis (labeled 2 and $2^{\prime}$ respectively in Fig. 4) can be obtained by the same method depicted above.

In any case, when $\beta>0$ and, $\gamma>0$ then light converges, toward the $x$ axis, otherwise $\beta<0$ and $\gamma>0$ means that light diverges from the $x$ axis.

\section{SIMULATION RESULTS OF EMITTING ANTENNA WITH ELLIPSOID-PARABOLOID SURFACE CONFIGURATION CONFOCAL SIMULATION OF ELLIPSOID-PARABOLOID SURFACES}

Suppose the focus of the parabola overlaps with the left focus of the ellipse, it means that the coordinate of the parabola apex is $P\left(-\frac{p}{2}-c, 0\right)$. Two-dimensional ray tracing simulation results, and the curves of the divergence angles of the edge emitting light are shown in Fig. 5.

In Fig. 5(a), color of lines from red to blue represent energy changes from high to low level. Fig. 5(b) shows that the divergence angles of inner and outer edge laser beam overlapped each other and all approach to 0 degrees, which means a precision collimated laser beam, which hopefully approaches the diffraction limit, will be produced by the confocal optical emitting antenna. This kind of novel antenna could greatly enhance the emission efficiency and collimation accuracy of optical antenna system for long range optical communication.

\subsection{On-axial and Off-axial Defocus Analysis of Ellipsoid- Paraboloid Surfaces}

Some mechanical assembly errors could cause the on-axial defocus of the ellipsoid-paraboloid mirror surfaces. And some thermal expansion damages may cause off-axial defocus of the two reflectors [10].

Suppose the amount of on-axial defocusing is $s$, and the off-axial defocusing amount is $t$, the coordinate of the parabola apex can be written as $P\left(-\frac{p}{2}-c+s, t\right)$. Twodimensional ray tracing simulation results and the curves of the divergence angles of the edge emitting light with change in on-axial defocusing amounts are shown in Fig. 6.

It has been presented in part II that $\beta>0$ and $\gamma>0$ mean that light converges toward the $x$ axis, otherwise $\beta<0$ and $\gamma>0$ mean that light diverges away from the $x$ axis. It can be inferred from Fig. 6 that the emitting light from the emitting antenna is convergent when the on-axial defocusing amount is negative (as shown in Fig. 6(a) and in the left half of Fig. 6(c)) and is divergence when the on-axial defocusing amount is positive (as shown in Fig. 6 


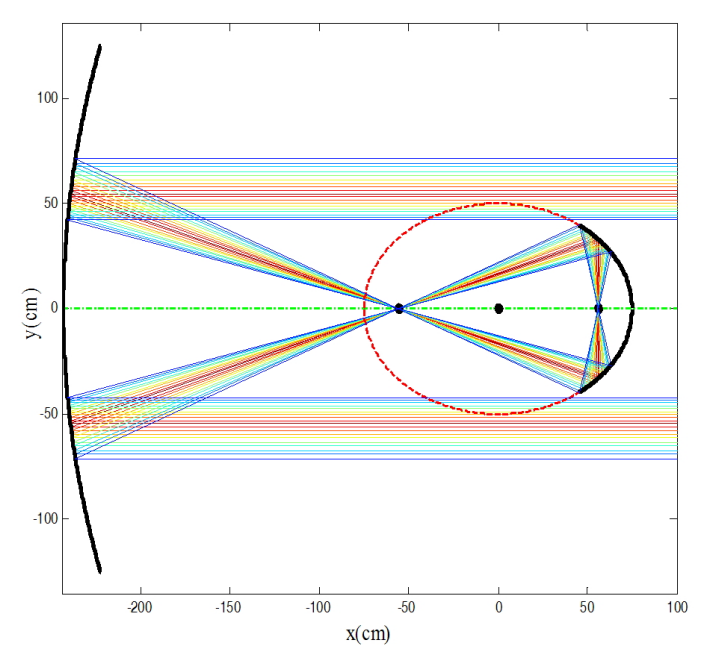

(a)

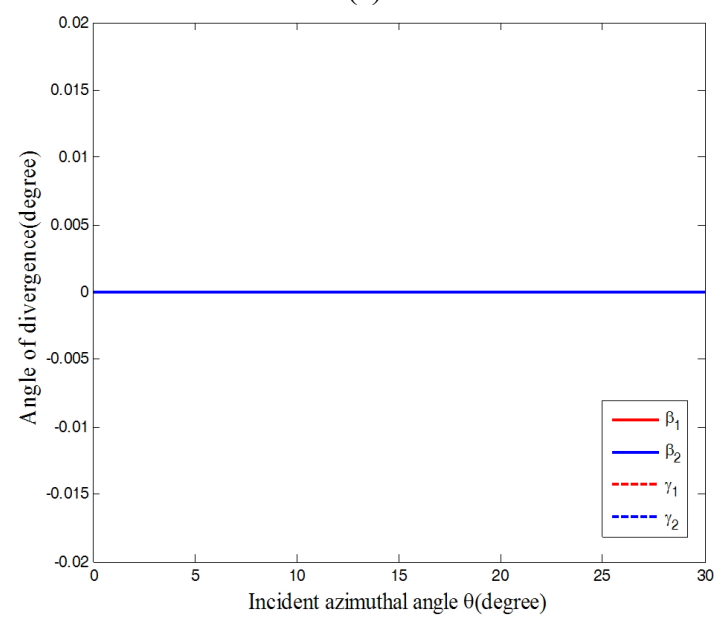

(b)

FIG. 5. Simulation results of the confocal optical emitting antenna, the coordinate of the parabola apex is $P\left(-\frac{p}{2}-c, 0\right)$, $p=375 \mathrm{~cm}, a=75 \mathrm{~cm}, b=50 \mathrm{~cm}$, and the azimuthal angle of the edge light emitted from the radial radiation fiber laser is $\theta=30^{\circ}$. (a) Two-dimensional ray tracing of the confocal optical emitting antenna. (b) The curves of the divergence angles of inner and outer edge laser beam emitted from confocal optical emitting antenna change with radial radiation azimuthal angle $\theta$, the observation plane is at position of $x=100 \mathrm{~cm}$.

(b) and in the right half of Fig. 6(c)).

Figure 7(a) and Fig. 7(b) shows two-dimensional ray tracing simulation results of an antenna with two different off-axial defocusing amounts. The emitting light tilts downward when the off-axial defocusing amount is $t=-17 \mathrm{~cm}$ (as shown in Fig. 7(a)) and tilts upward when the off-axial defocusing amount is $t=17 \mathrm{~cm}$ (as shown in Fig. 7(b)). In this time, $\beta$ and $\gamma$ are regarded as the deviation angles between the tilted emitting light and the $x$ axis, as shown in Fig. 7(c).

It can be seen from Fig. 7(c) that when the off-axial

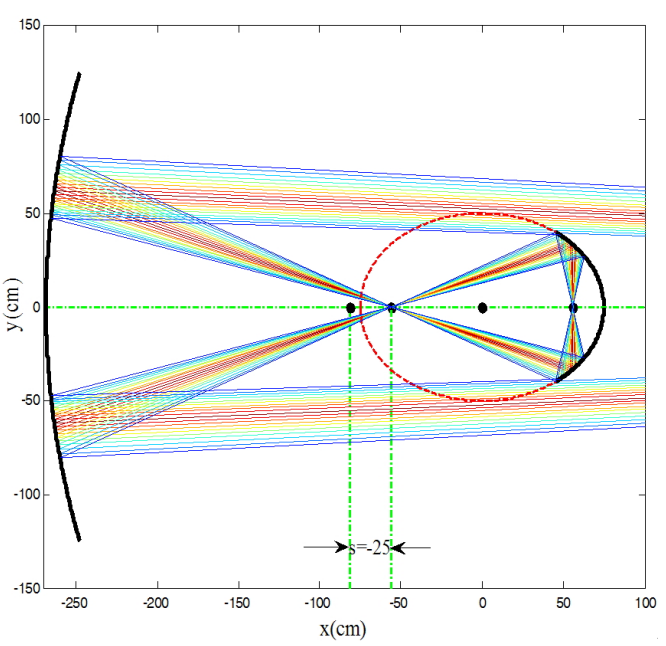

(a)

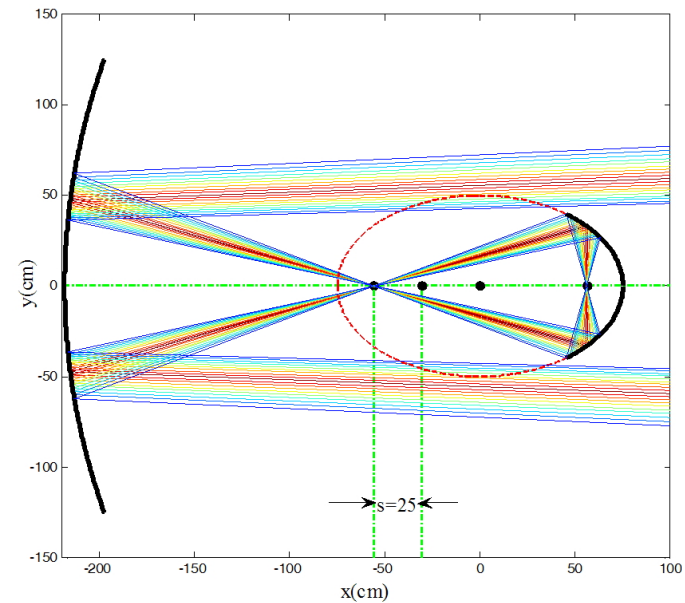

(b)

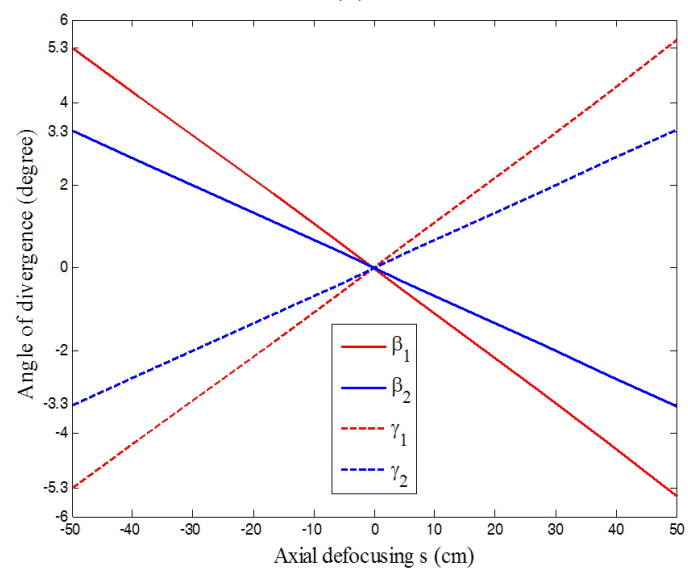

(c)

FIG. 6. Two-dimensional ray tracing simulation results and curves of the divergence angles of the edge emitting ray from emitting antenna models vs. different on-axial defocusing amounts. The observation plane is at position of $x=100 \mathrm{~cm}$. (a) Two-dimensional ray tracing simulation result of on-axial defocusing amount $s=-25 \mathrm{~cm}$ and (b) on-axial defocusing amount $s=25 \mathrm{~cm}$, (c) the curves of the divergence angles of the edge emitting light vs. on-axial defocusing amount $s$ changes from $-50 \mathrm{~cm}$ to $50 \mathrm{~cm}$. 


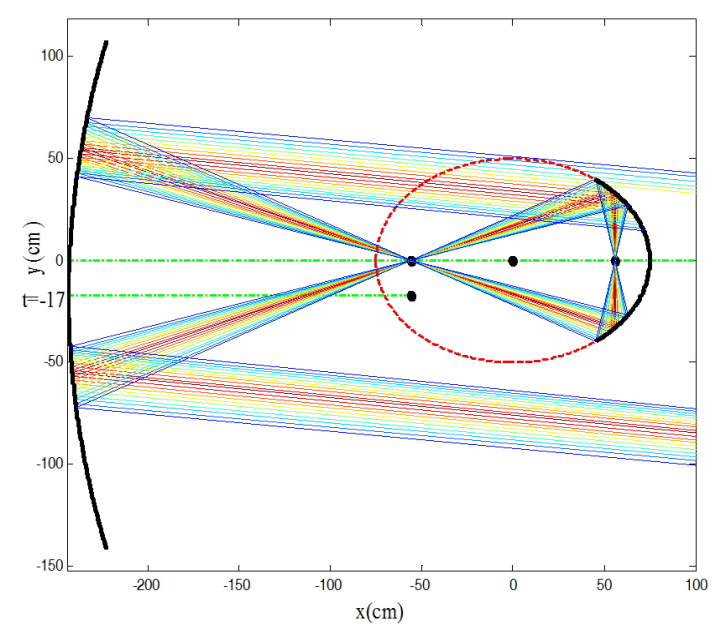

(a)

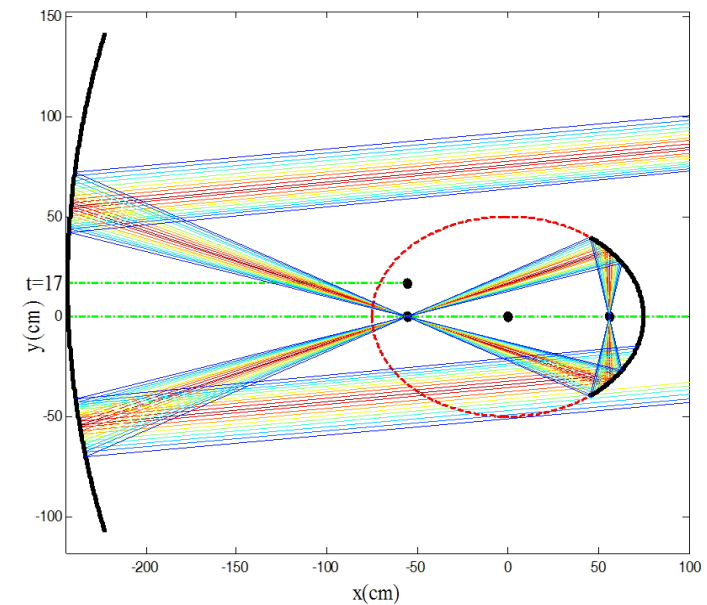

(b)

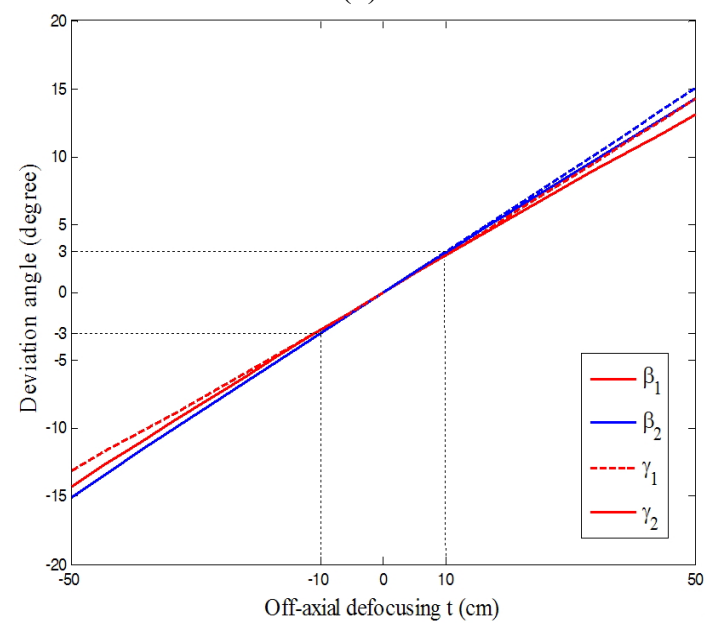

(c)

FIG. 7. Two-dimensional ray tracing simulation results and curves of the deviation angles of the edge emitting light of emitting antenna models vs. different off-axial defocusing amounts. (a) Two-dimensional ray tracing simulation result of off-axial defocusing amount $t=-17 \mathrm{~cm}$ and (b) off-axial defocusing amount $t=17 \mathrm{~cm}$, (c) the curves of the deviation angles of the edge emitting light vs. off-axial defocusing amount $t$ change from $-50 \mathrm{~cm}$ to $50 \mathrm{~cm}$. defocusing $t$ is between $-10 \mathrm{~cm}$ and $10 \mathrm{~cm}$, curves of deviation angles vs. off-axial defocusing almost overlapped, from which it can be inferred that the tilted emitting light is still collimated when the off-axial defocusing $t$ is in this range. It also can be inferred from Fig. 6 and Fig. 7 that, light blocking by the secondary mirror occurs in the defocused antenna, which causes energy loss of the emitting antenna system. Figure 8 shows the curves of emission efficiency of emitting antenna vs. different on-axial and off-axial defocusing amounts.

The curve for the on-axial defocusing presented by the solid line in Fig. 8(a), when the on-axial defocusing amount $s$ is negative, the larger absolute value of on -axial defocusing is, the greater divergent emitting light there is. Because of the secondary mirror blocking, the emission efficiency of antenna monotonically decreases with increase of the absolute value of the on-axial defocusing. If the on-axial defocusing is

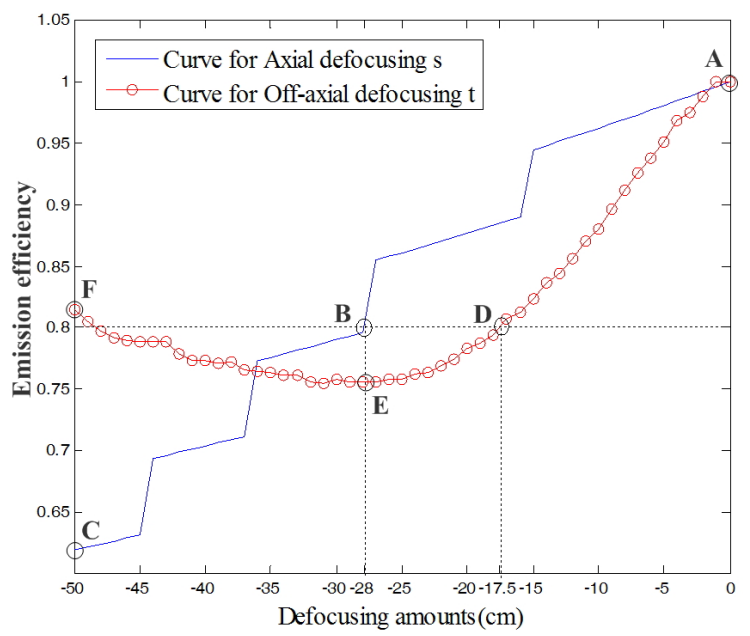

(a)

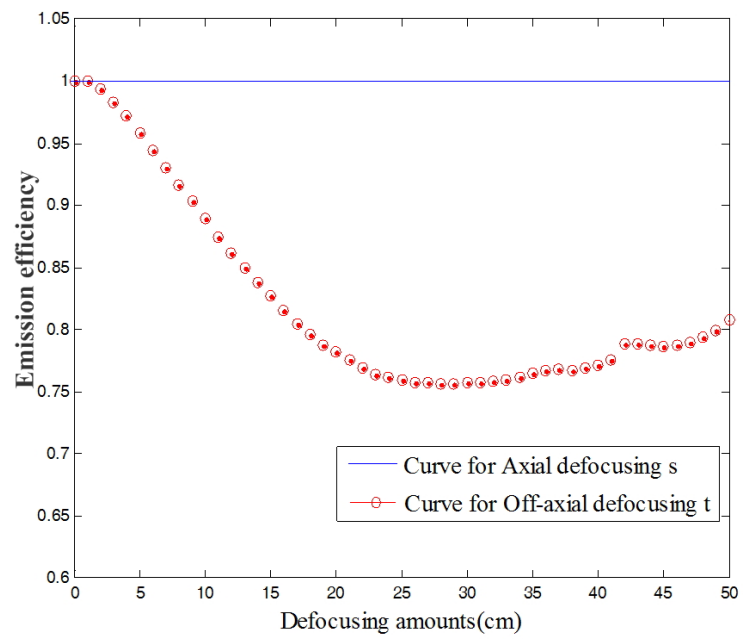

(b)

FIG. 8. Curves for defocusing amounts vs. emission efficiency of emitting antenna. (a) Curves of negative defocusing amounts vs. emission efficiency. (b) Curves of positive defocusing amounts vs. emission efficiency. 
positive, no blocking of the secondary mirror on the divergent emitting light, emission efficiency remains $100 \%$, as shown by solid line in Fig. 8(b).

For the off-axial defocus case, the emitting light tilts downward when the off-axial defocusing amount $t$ is negative and tilts upward when the $t$ is positive, the secondary mirror blocking always exists. With increase of the absolute value of off-axial defocusing, emission efficiency is gradually reduced. When the secondary mirror blocking is bypassed, the emission efficiency gradually increases once again. Curve of negative off-axial defocusing amounts vs. emission efficiency (as shown by circle line in Fig. 8(a)) is symmetrically distributed compare with the curve of positive off-axial defocusing amounts (as shown by circle line in Fig. 8(b)). When the on-axial and off-axial defocusing amounts are $-28 \mathrm{~cm}$ and $-17.5 \mathrm{~cm}$, respectively, the emission efficiency reduced to $80 \%$. When the off-axial defocusing amount is $-28 \mathrm{~cm}$, the emission efficiency reduced to minimum $75 \%$.

Figure 9 shows spot diagrams with different defocusing amounts, obtained at the plane positioned at $x=100 \mathrm{~cm}$.

When the confocal condition is satisfied, transmitting light is a high-precision collimated hollow beam, and just in time to avoid the secondary mirror blocking (as shown in Fig. 9(a)). The emission efficiency is $100 \%$ (corresponding to point A in Fig. 8(a)). For the case of negative on-axial defocusing amounts, i.e. $s<0$, emitting light is convergent, the inner boundary of the light is blocked by the secondary mirror. When $s=-28 \mathrm{~cm}$ (as shown in Fig. 9(b)), the emission efficiency approaches to $80 \%$ (corresponding to point B in Fig. 8(a)). When $s=-50 \mathrm{~cm}$ (as shown in Fig. 9(c)), the emission efficiency reduces to $60 \%$ (corresponding to point $\mathrm{C}$ in Fig. 8(a)). For the case of $t<0$, light tilts downward, but the collimation degree is almost unchanged, serious energy loss is caused by the secondary mirror blocking. When $t=-17.5 \mathrm{~cm}$ (as shown in Fig. 9(d)), the secondary mirror blocking leads to emission efficiency reduction approaching $80 \%$ (corresponding to point D in Fig. 8(a)). When $t=-28 \mathrm{~cm}$ (as shown in Fig. 9(e)), emission efficiency reduces to $75 \%$ (corresponding to point $\mathrm{E}$ in Fig. 8(a)). Afterwards, the area of the secondary mirror blocking is reduced, and the emission efficiency increases. When $t=-50 \mathrm{~cm}$ (as shown in Fig. 9(f)), emission efficiency approaches $82 \%$ (corresponding to point $\mathrm{F}$ in Fig. 8(a)), but the beam has deviated from the $x$ axis by 5.3 degrees (as shown in Fig. 7(c)), long range optical communication cannot be realized. The spot diagrams of negative off-axial defocusing amounts are symmetrically distributed as those of the positive off-axial defocusing amounts.

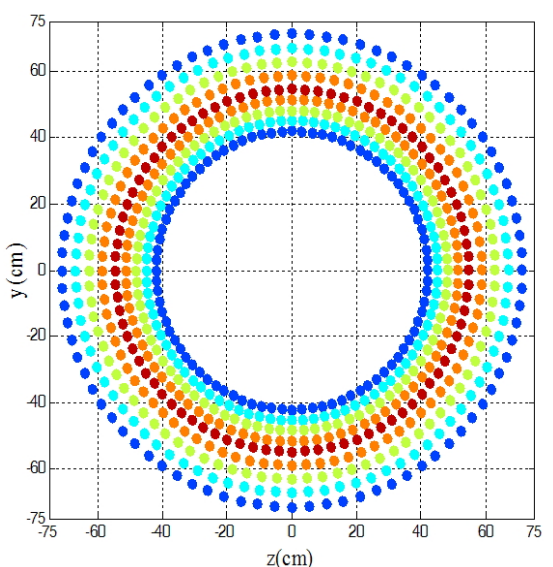

(a)

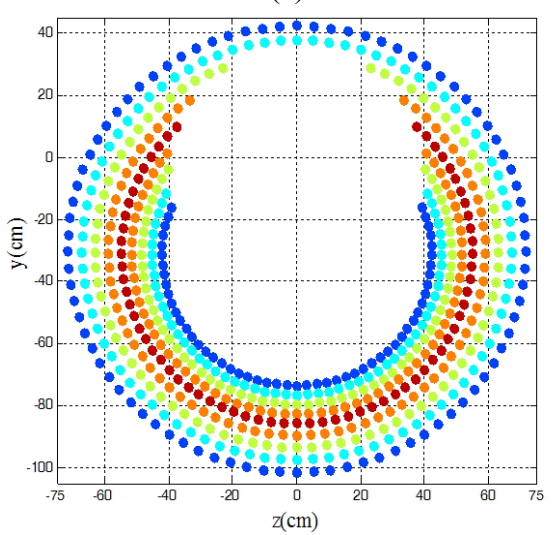

(d)

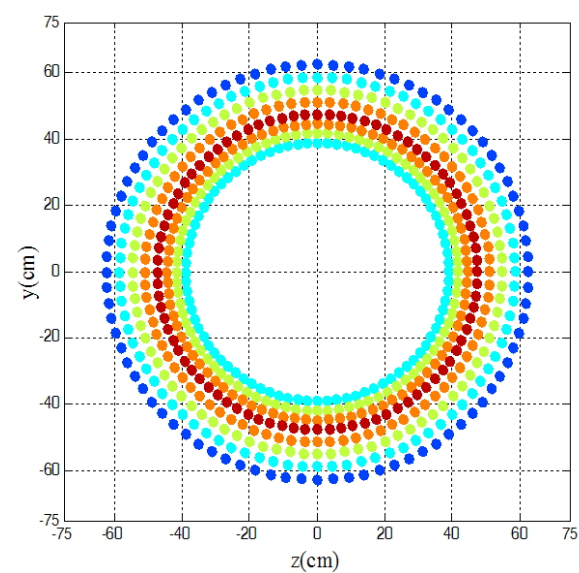

(b)

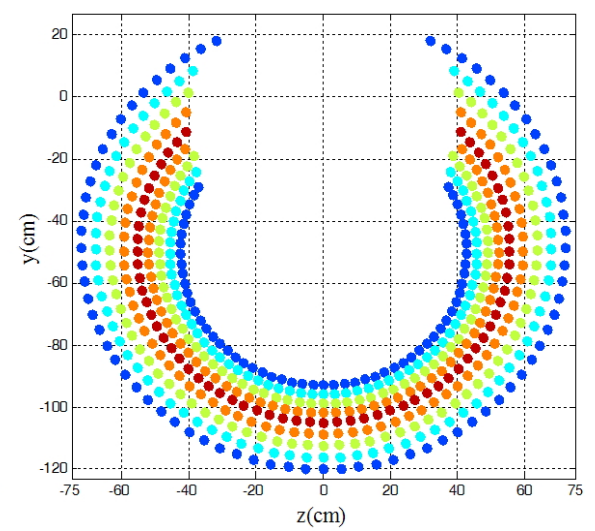

(e)

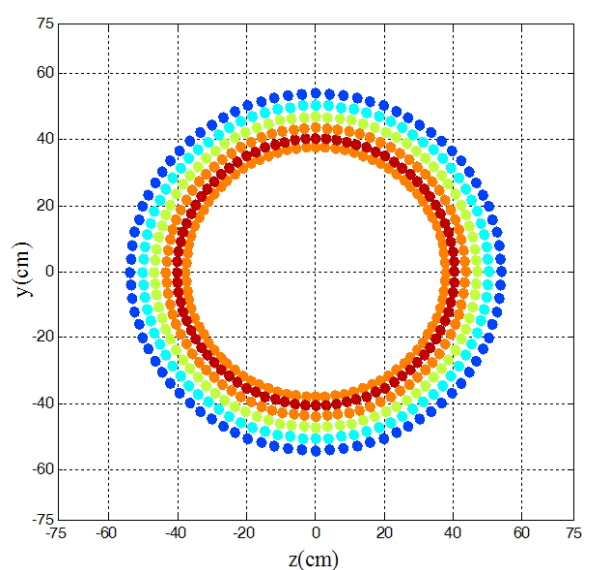

(c)

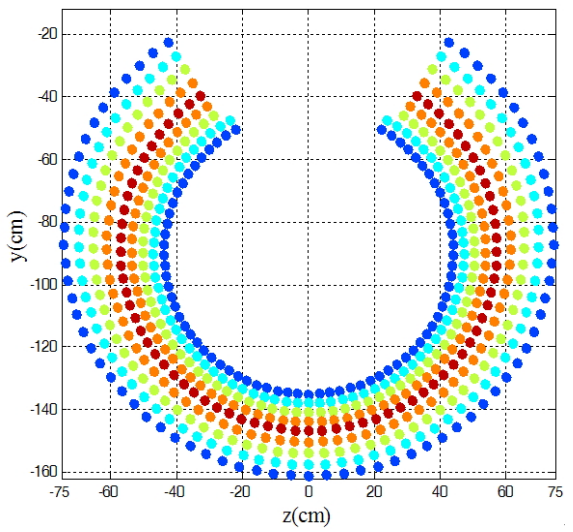

(f)

FIG. 9. Spot diagrams with different defocusing amounts. (a) On-axial defocusing amount is $s=0$, (b) $s=-28 \mathrm{~cm}$, (c) $s=-50 \mathrm{~cm}$. (d) Off-axial defocusing amount is $t=-17.5 \mathrm{~cm}$, (e) $t=-28 \mathrm{~cm}$, (f) $t=-50 \mathrm{~cm}$. 


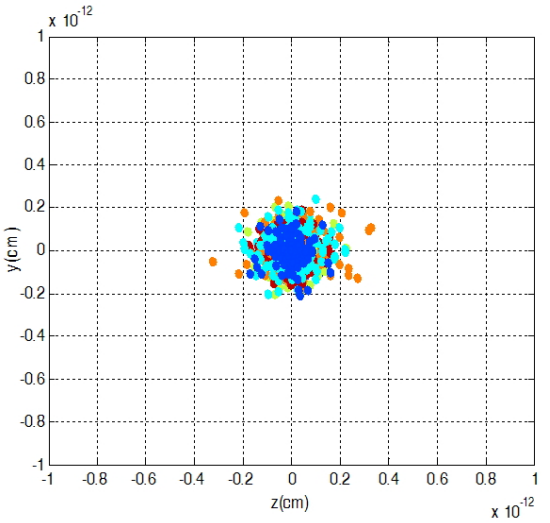

(a)

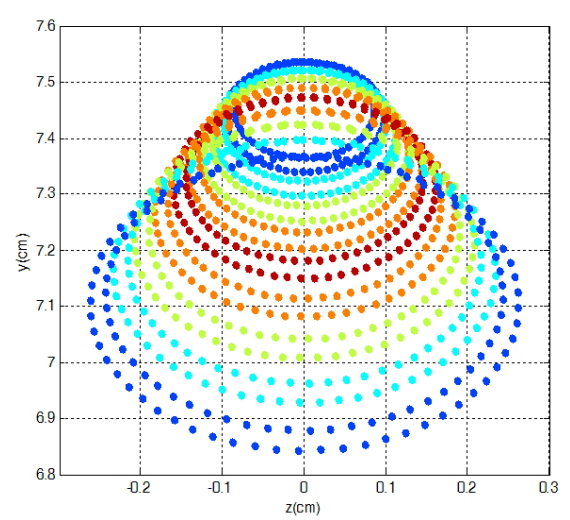

(b)

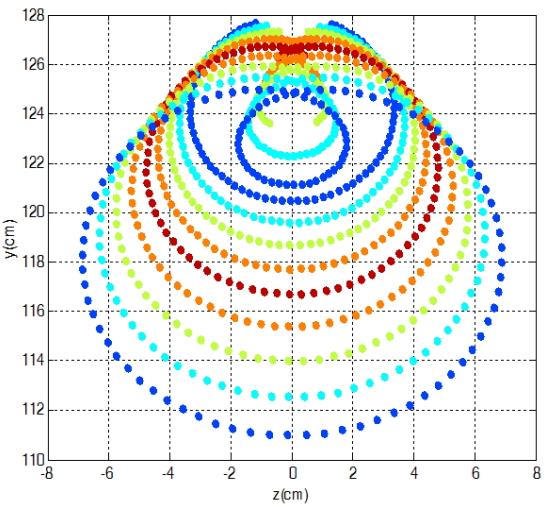

(c)

FIG. 10. Simulation results of emitting antenna aberration. (a) Spot diagram at the Gaussian plane for the confocal case and (b) coma aberration of emitting antenna with off-axial defocusing amount is $t=1 \mathrm{~cm}$, (c) coma and astigmatism aberrations of emitting antenna with off-axial defocusing amount is $t=17 \mathrm{~cm}$.

By introducing of an berration-free ideal converging lens at the exit pupil of the emitting antenna, optical aberration can be exactly visible at its image plane. Figure 10 shows spot diagrams obtained at the Gaussian plane of the ideal converging lens.

For the confocal case, spot diameter at the Gaussian plane of the ideal converging lens is approximately $0.4 \times 10^{-12}$ (as shown in Fig. 10(a)), the confocal antenna can be regarded as an aberration-free system. Obviously, convergent or divergent output beam caused by on-axial defocusing may shows spherical aberration at the Gaussian plane. For the off-axial defocusing case, small off-axial defocusing amount cause coma aberration (as shown in Fig. 10(b)), too large off-axial defocusing amounts may terminate coma and astigmatism aberrations at same time (as shown in Fig. 10(c)). Thus the smaller the amount of defocusing is, the higher the optical quality of the antenna system is. According to the Strehl criterion, when the receiving efficiency is greater than $80 \%$, the imaging quality of the optical system can be regarded as perfect.

In order to further study the light transmission efficiency in the whole optical communication system, we must not only consider the impact of defocusing amounts and aberrations, but also consider the impact of the transmission distance on the receiving efficiency of the receiving antenna.

\section{SIMULATION RESULTS OF RECEIVING ANTENNA}

A receiving antenna always uses a typical Cassegrain telescope as shown in Fig. 11(a). The apertures of the primary mirror and secondary mirror are $\Phi=150 \mathrm{~mm}$ and $\phi=30 \mathrm{~mm}$, respectively. The obscuration ratio is $\phi / \Phi=0.2$. The effective receiving plane can be considered as a concentric ring with outer and inner radii of $150 \mathrm{~mm}$ and $30 \mathrm{~mm}$, respectively, as shown in Fig. 11(b).

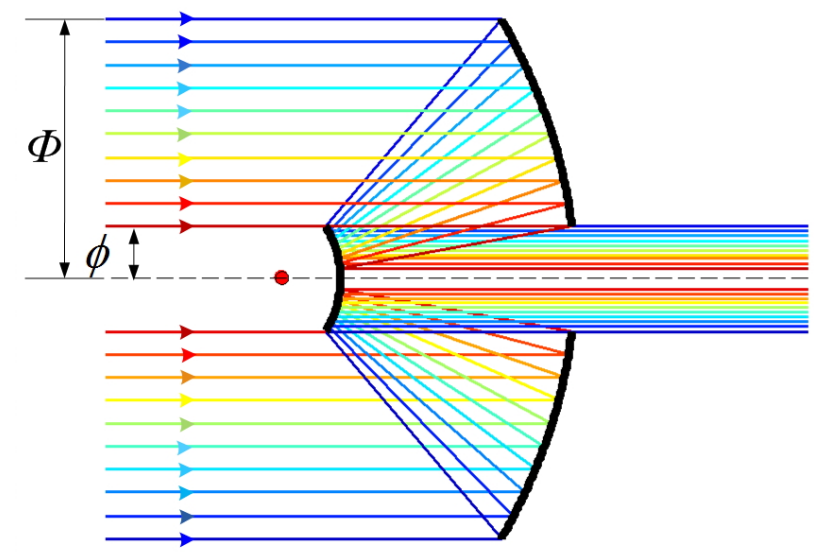

(a)

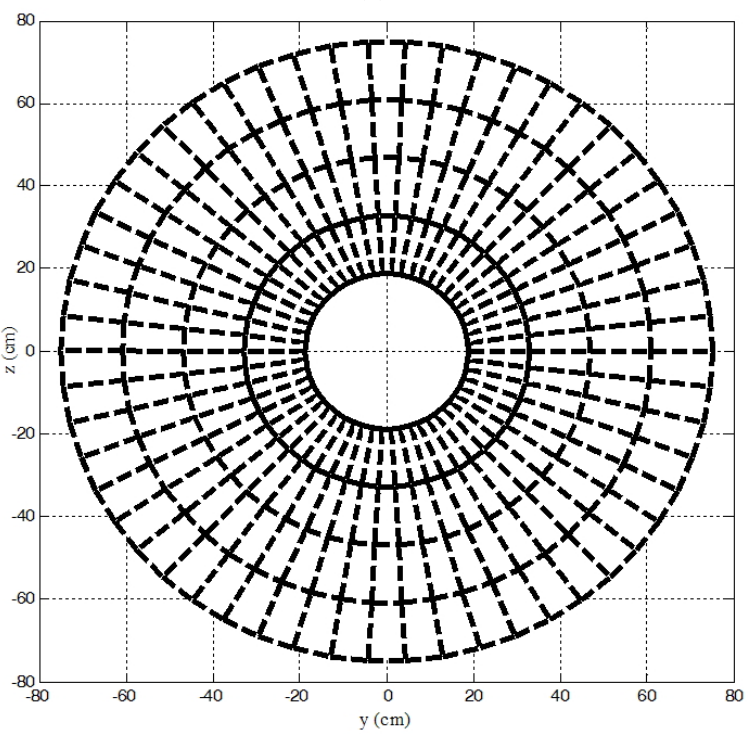

(b)

FIG. 11. Schematic diagram of Cassegrain antenna and effective receiving plane. (a) Structure of receiving antenna and (b) effective receiving plane. 
Figure 12 shows the spot diagrams on the effective receiving plane, which is a distances of $5000 \mathrm{~m}$ from the emitting antenna.

In Fig. 12(a), when $s=-0.5 \mathrm{~mm}$, the spot diagram at the effective receiving plane is concentrated near the inner ring edge. The inner part of hollow beam energy is blocked by the secondary mirror of the receiving antenna. In Fig. 12(b), when $s=0 \mathrm{~mm}$, a precision collimated laser beam is completely received by the effective receiving plane. In Fig. 12(c), when $s=0.2 \mathrm{~mm}$, the spot diagram at the receiving plane is near the outer ring edge, the outer edge of the hollow beam failed to enter the effective receiving plane. It can be seen that, positive on-axial defocusing (i.e. $s>0$ ) has greater influence on the receiving efficiency than the negative on-axial defocusing. From Fig. 12(d) to Fig. 12(f), the off-axial defocusing $t$ changes from $0.1 \mathrm{~mm}$ to $0.5 \mathrm{~mm}$, the spot diagram at the effective receiving plane moves upward, part of the light is blocked by the secondary mirror of the receiving antenna. By integral calculation of spot energy at the effective receiving plane, and comparison with emitting energy of confocal emitting antenna, the relationship between energy loss of the optical antenna system and transmission distance $L$ with different defocusing amounts could be obtained, as shown in Fig. 13.
It can be inferred from Fig. 13(a), that when the on-axial defocusing is $s=0.1 \mathrm{~mm}$, the maximum transmission distance achieves $L=5000 \mathrm{~m}$ and the receiving antenna efficiency is reduced to $80 \%$. From Fig. 13(b), when off-axial defocusing is $t=0.1 \mathrm{~mm}$, the maximum transmission distance achieves $L=4680 \mathrm{~m}$ and the receiving efficiency is reducesd to $80 \%$. According to the Strehl criterion, to realize long range optical communication $(L>5000 \mathrm{~m})$, the on-axial defocusing and off-axial defocusing must be less than 0.1 $\mathrm{mm}$, which could be caused by thermal expansion damages or mechanical assembly errors of the emitting antenna.

It can be also inferred that curves shown in Fig. 13(a) present linear distribution, and curves shown in Fig. 13(b) present nonlinear distribution, which means the effects of off-axial defocusing on the receiving efficiency is greater than that of the on-axial defocusing. Curves of off-axial defocusing $t$ vs. receiving efficiency at different transmission distances are shown in Fig. 14.

It can be inferred from Fig.14 that for long transmission distance $L>100 \mathrm{~km}$, curves of off-axial defocusing in range of $-0.05 \mathrm{~mm}<t<0.05 \mathrm{~mm}$ have the same distribution. When the off-axial defocusing is in the range of $-0.01 \mathrm{~mm}$ $<t<0.01 \mathrm{~mm}$, the receiving efficiency is higher than $80 \%$, long range optical communication could be guaranteed.

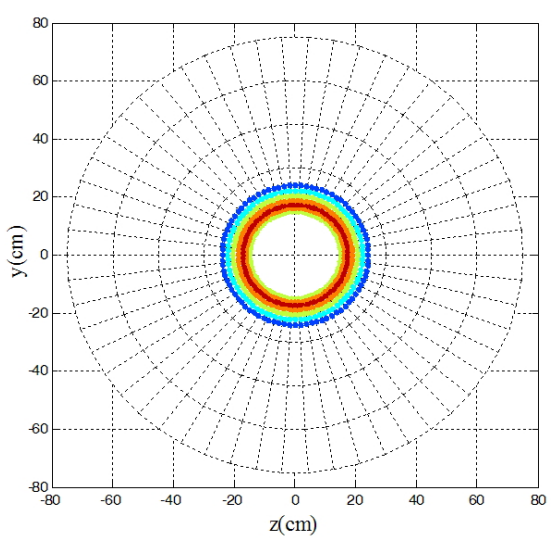

(a)

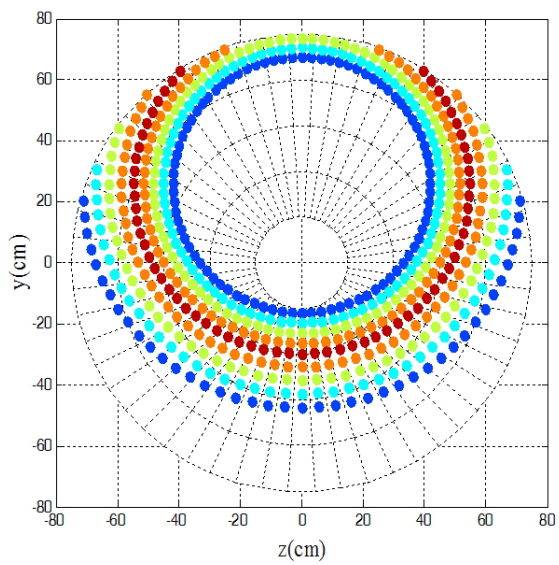

(d)

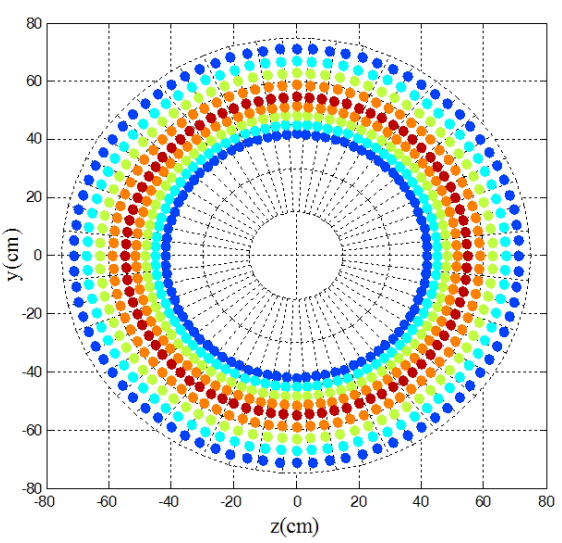

(b)

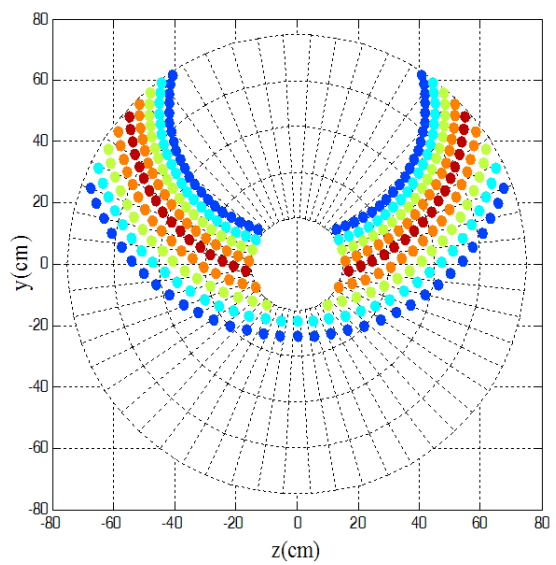

(e)

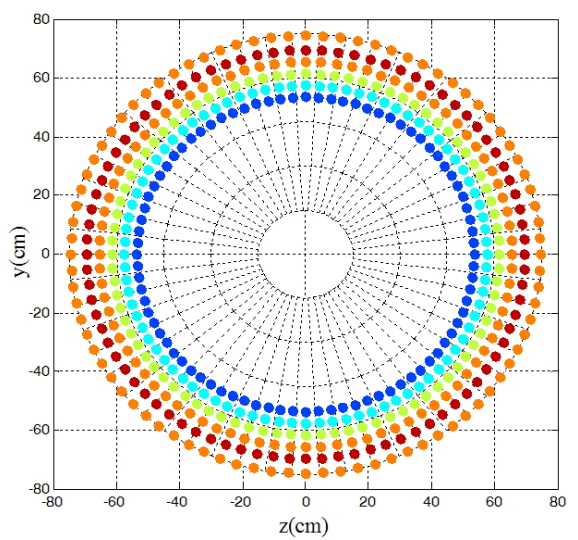

(c)

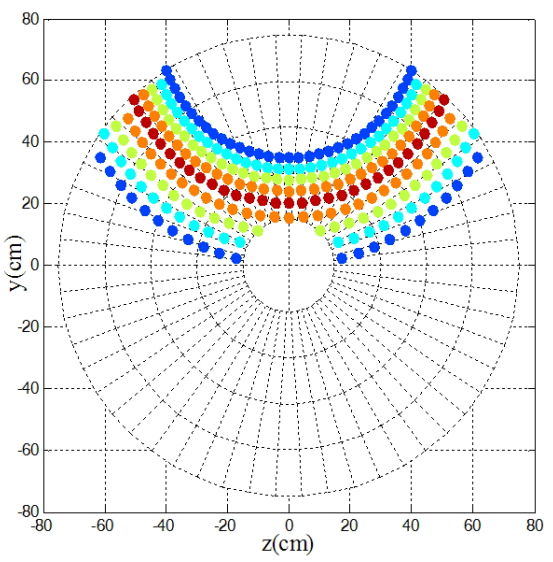

(f)

FIG. 12. Spot diagram on the effective receiving plane at $x=5000 \mathrm{~m}$ with different defocusing amounts, (a) $s=-0.5 \mathrm{~mm}$ (b) $s=0 \mathrm{~mm}$, (c) $s=0.2 \mathrm{~mm}$, (d) $t=0.1 \mathrm{~mm}$, (e) $t=0.2 \mathrm{~mm}$, (f) $t=0.3 \mathrm{~mm}$. 


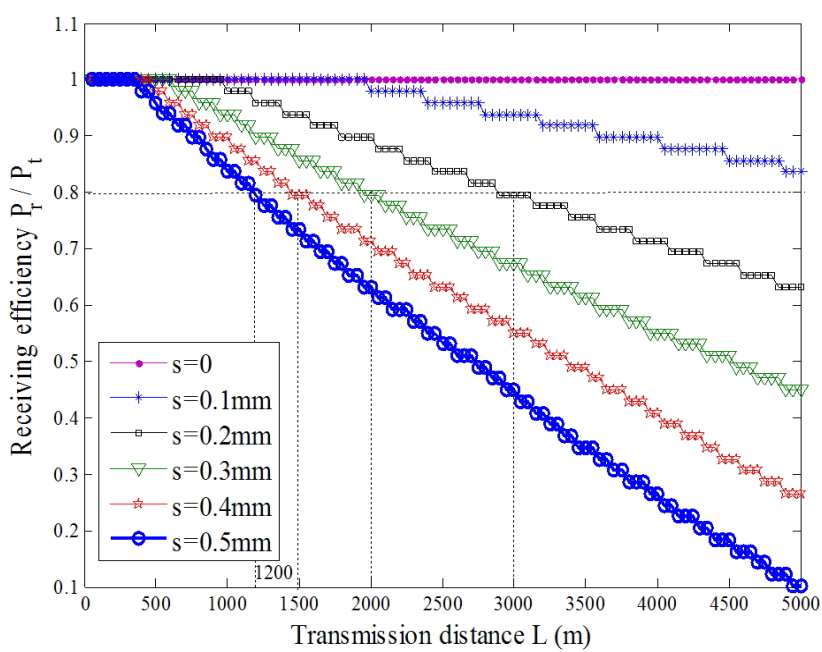

(a)

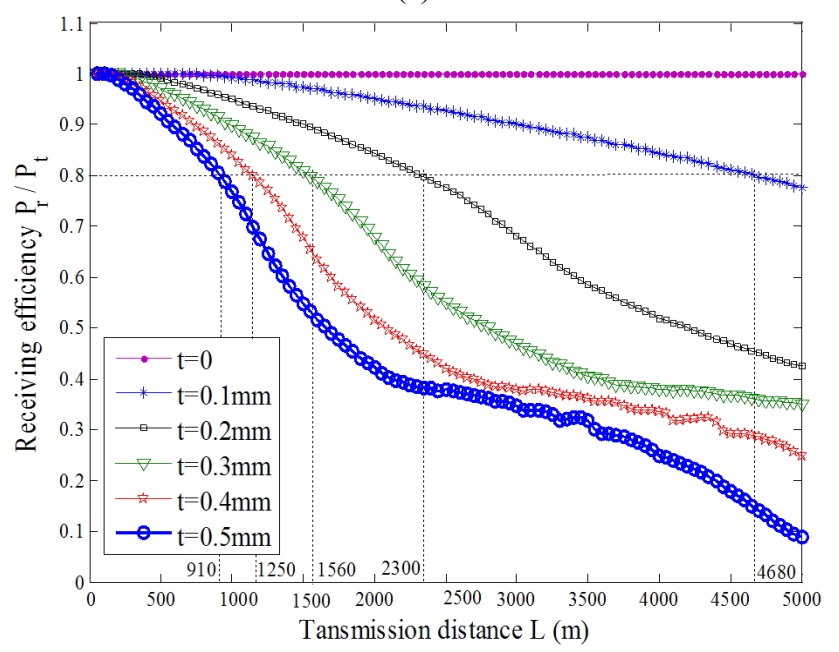

(b)

FIG. 13. Relationship between receiving efficiency and transmission distance $L$. (a) Curves of receiving efficiency vs. transmission distance $L$ with different on-axial defocusing $s$, (b) curves of receiving efficiency vs. transmission distance $L$ with different off-axial defocusing $t$.

\section{CONCLUSION}

A novel antenna with ellipsoid-paraboloid surfaces configuration is designed for matching the incident radial radiation fiber laser distribution for maximum transmission efficiency. It can be inferred from simulation results that the effects of off-axial defocusing between the primary and secondary mirrors of emitting antenna on the receiving efficiency is greater than that of the on-axial defocusing. When the absolute value of off-axial defocusing is less than 0.05 $\mathrm{mm}$, curves of receiving efficiency for long transmission distance $L>100 \mathrm{~km}$ have the same distribution. When the absolute value of off-axial defocusing is less than $0.01 \mathrm{~mm}$, the receiving efficiency is higher than $80 \%$, long range optical communication could be guaranteed.

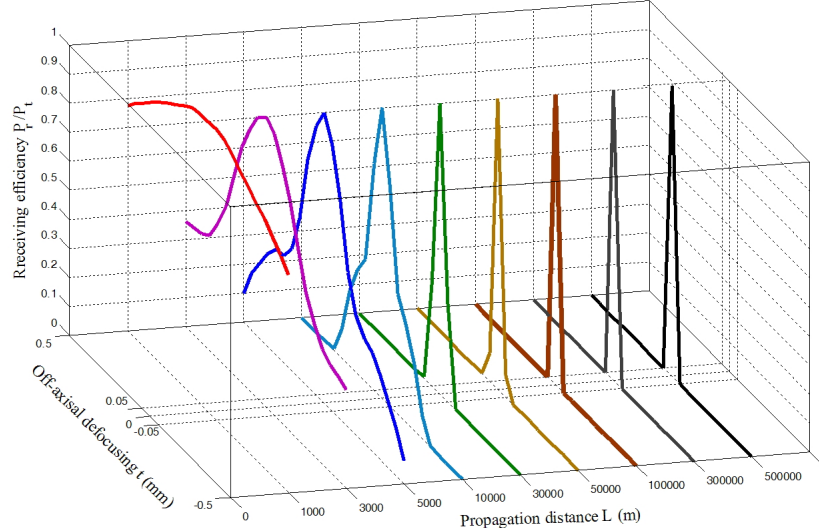

(a)

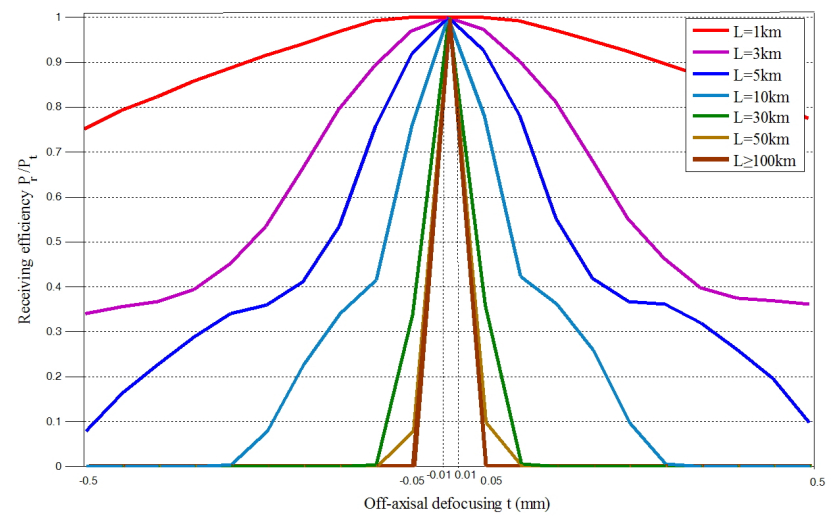

(b)

FIG. 14. Curves of off-axial defocusing $t$ vs. receiving efficiency at different transmission distance $L$ in long range optical communication, (a) three-dimensional distribution (b) two-dimensional distribution.

\section{ACKNOWLEDGMENT}

This work is supported by the National Natural Science Foundation of China under Grant No. 61271167 and No. 61307093, and also supported by Research Foundation of the General Armament Department of China under Grant No. 9140A07040913DZ02106 and the Fundamental Research Funds for the Central Universities under Grant No. ZYGX2013J051.

\section{REFERENCES}

1. Y. M. Duan, H. J. Yang, P. Jiang, and P. Wang, "Research on the solar concentrating optical system for solar energy utilization," J. Opt. Soc. Korea 17, 371-375 (2013).

2. S. Choi, "New type of white-light LED lighting for illumination and optical wireless communication under obstacles," J. Opt. Soc. Korea 16, 203-209 (2012).

3. Z. H. Chen, H. J. Yang, X. Y. Wang, J. Wang, and X. P. Huang, "Theoretical analysis and test for off-axisal Cassegrain optical antenna,” Int. J. Light Elect. Opt. 123, 
268-271 (2011).

4. S. D. Jackson, "Towards high-power mid-infrared emission from a fibre laser," Nat. Photonics 6, 423-431 (2012).

5. S. K. Turitsyn, S. A. Babin, A. E. El-Taher, P. Harper, D. V. Churkin, S. I. Kablukov, J. D. Ania-Castañón, V. Karalekas, and E. V. Podivilov, "Random distributed feedback fibre laser," Nat. Photonics 4, 231-235 (2012).

6. C. Jauregui, J. Limpert, and A. Tünnermann, "High-power fibre lasers," Nat. Photonics 7, 681-867 (2013).

7. M. E. Fermann and I. Hartl, "Ultrafast fibre lasers," Nat. Photonics 7, 868-874 (2013).
8. A. A. Fotiadi and P. Mégre, "Surface-emiting fibre lasers: Perfect ring-like beam," Nat. Photonics 6, 217-219 (2012).

9. A. M. Stolyarov, L. Wei, O. Shapira, F. Sorin, S. L. Chua, J. D. Joannopoulos, and Y. Fink, "Microfluidic directional emission control of an azimuthally polarized radial fibre laser," Nat. Photonics 6, 229-233 (2012).

10. H. Sun, H. J. Yang, Y. Peng, X. L. Li, and S. S. Li, "3-D simulation research for off-axisal Cassegrain optical antenna and coupling systems," Optoelectr. Adv. Mat. 6, 284-287 (2012). 